\title{
Metrisability of three-dimensional path geometries
}

\author{
Maciej Dunajski $^{1} \cdot$ Michael Eastwood $^{2}$
}

Received: 1 December 2014 / Revised: 21 May 2015 / Accepted: 19 November 2015 /

Published online: 8 March 2016

(C) Springer International Publishing AG 2016

\begin{abstract}
Given a projective structure on a three-dimensional manifold, we find explicit obstructions to the local existence of a Levi-Civita connection in the projective class. These obstructions are given by projectively invariant tensors algebraically constructed from the projective Weyl curvature. We show, by examples, that their vanishing is necessary but not sufficient for local metrisability.
\end{abstract}

Keywords Projective differential geometry · Path geometry · Weyl geometry • Metrisability

Mathematics Subject Classification 53A20

\section{Introduction}

There are several inequivalent geometric structures that give rise to a preferred family of curves on a smooth $n$-manifold $M$. A path geometry on $M$ is a locally defined family of unparametrised smooth curves (called paths), one through each point and in each direction. A path geometry is projective if its paths are the unparametrised geodesics of a torsion-free connection $\nabla$ on $T M$. The corresponding projective structure $(M,[\nabla])$ is then defined by the equivalence class of torsion-free connections sharing

Michael Eastwood

meastwoo@member.ams.org

Maciej Dunajski

m.dunajski@damtp.cam.ac.uk

1 Department of Applied Mathematics and Theoretical Physics, University of Cambridge,

Wilberforce Road, Cambridge CB3 0WA, UK

2 School of Mathematical Sciences, University of Adelaide, SA 5005, Australia 
their unparametrised geodesics with $\nabla$. Finally a path geometry is metrisable if its paths are the unparametrised geodesics of a (pseudo-)Riemannian metric $g$. In this case the path geometry is, of course, also projective since the underlying projective structure is defined by the Levi-Civita connection of $g$. The converse does not hold: a general projective structure does not contain a Levi-Civita connection of any metric.

The characterisation of metrisable projective structures is a classical problem, which goes back to the work of Roger Liouville [17]. This problem has recently been solved if $n=2$ : in this case (assuming real-analyticity for sufficiency, as one must) necessary and sufficient conditions are given by the vanishing of a set of projective differential invariants [2], the simplest of which is of differential order five in the connection coefficients of a chosen $\nabla \in[\nabla]$. The case $n=2$ is special-the projective Weyl curvature vanishes on a surface. This is no longer the case if $n=3$, where the first set of obstructions already arises at order one, and is algebraic in the projective Weyl tensor. In this paper we shall present some of these obstructions as explicit projectively invariant tensors constructed algebraically from the Weyl curvature.

As is often done in differential geometry, we shall adorn tensors with indices in order to denote the type of the tensor. Thus, we may denote a vector or vector field by $X^{a}$ but $\omega_{a}$ always denotes a co-vector or 1-form. This is Penrose's abstract index notation [22]. The canonical pairing between vectors and co-vectors is denoted by repeating an index so that $X^{a} \omega_{a}$ is the scalar that would often be written without indices as $X\lrcorner \omega$. For any tensor $\psi_{a b c}$, we shall denote its skew part by $\psi_{[a b c]}$ and its symmetric part by $\psi_{(a b c)}$.

Projective structures are reviewed at the start of Sect. 2. Here, suffice it to say that the primary invariant of a projective structure is the projective Weyl tensor $W_{a b}{ }^{c} d$, an irreducible part of the curvature of any connection in the projective class [ $\nabla]$ satisfying

$$
W_{a b}^{c} d=W_{[a b]}^{c} d, \quad W_{\left[a b^{c} d\right]}^{c}=0, \quad W_{a b}^{a} d=0 .
$$

This article is concerned exclusively with the 3-dimensional case. To formulate one of our results define a traceless tensor $V^{a b}{ }_{c}=V^{(a b)}{ }_{c}$ in terms of the projective Weyl curvature $W_{a b}{ }^{c} d$ and an arbitrarily chosen non-degenerate section $\epsilon^{a b c}$ of $\Lambda^{3}(T M)$ by

$$
V_{c}^{a b}=\epsilon^{d e a} W_{d e}{ }^{b}{ }_{c} .
$$

Theorem 1.1 Let $A, B, C, D, F, J, K, L$ be the symmetric tensors defined by

$$
\begin{aligned}
A^{a b} & =\bigodot\left(V^{a p}{ }_{q} V^{b q}{ }_{p}\right), \\
B^{a b c} & =\bigodot\left(V^{a p}{ }_{q} V^{b q}{ }_{r} V^{c r}{ }_{p}\right), \\
C^{a b c} & =\bigodot\left(V^{a b}{ }_{p} V^{p q_{r}} V^{c r}{ }_{q}\right), \\
D^{a b c d} & =\bigodot\left(V^{a b}{ }_{p} V^{p q_{r}} V^{c r}{ }_{s} V^{d s}{ }_{q}\right), \\
J^{a b c d e f} & =\bigodot\left(V^{a b}{ }_{p} V^{c d}{ }_{q} V^{p q}{ }_{r} V^{e r}{ }_{s} V^{s t}{ }_{u} V^{f u}{ }_{t}\right), \\
F^{a b c d} & =\bigodot\left(V^{a b}{ }_{p} V^{c d}{ }_{q} V^{p r}{ }_{s} V^{q s}{ }_{r}\right), \\
K^{a b c d e f} & =\bigodot\left(V^{a b}{ }_{p} V^{c d}{ }_{q} V^{e p}{ }_{r} V^{f q}{ }_{s} V^{r t}{ }_{u} V^{s u}{ }_{t}\right),
\end{aligned}
$$




$$
L^{a b c d e f}=\bigodot\left(V_{p}^{a b} V^{c d}{ }_{q} V^{p q}{ }_{r} V^{r s}{ }_{t} V^{e t}{ }_{u}{ }^{f u}{ }_{s}\right),
$$

where $\odot$ denotes symmetrisation over the non-contracted indices, and let

$$
T=24 J+12 K-24 L-24 B \odot B-24 C \odot C+40 B \odot C-24 A \odot D+6 A \odot F .
$$

In general, the tensor $T^{\text {abcdef }}$ does not vanish. However, if $\nabla$ is projectively equivalent to a Levi-Civita connection then $T^{\text {abcdef }} \equiv 0$.

Theorem 1.2 With tensors $A^{a b}, B^{a b c}, C^{a b c}, D^{a b c d}, F^{a b c d}, J^{a b c d e f}, K^{a b c d e f}, L^{a b c d e f}$ defined as in Theorem 1.1, the following

$$
\begin{array}{ll}
C^{a b c}-2 B^{a b c}, & F^{a b c d}-2 D^{a b c d}, \quad J^{a b c d e f}-2 L^{a b c d e f}, \\
3 J^{a b c d e f}-2 C^{(a b c} C^{d e f)}, & J^{a b c d e f}-4 K^{a b c d e f}+4 A^{(a b} D^{c d e f)}
\end{array}
$$

are generally non-zero but vanish if $\nabla$ is projectively equivalent to a Levi-Civita connection.

In fact, since

$$
\begin{aligned}
T=12(J-2 L) & -3(J-4 K+4 A \odot D)+5(3 J-2 C \odot C) \\
+ & 2(C-2 B) \odot(6 B-7 C)+6 A \odot(F-2 D),
\end{aligned}
$$

Theorem 1.2 implies Theorem 1.1. However, we shall see that the invariant $T$ arises in a more fundamental way, already described in [2]. More specifically, one can construct from the Weyl curvature a homomorphism $\Xi: \bigodot^{2}(T M) \rightarrow \bigodot^{3}(T M)$ that must have a non-trivial kernel in the metrisable case and $T^{\text {abcdef }}$ is defined to be what amounts to the determinant of this homomorphism: $T^{a b c d e f} X_{a} X_{b} X_{c} X_{d} X_{e} X_{f}$ is characterised as being the determinant of the composition

$$
\bigodot^{2}(T M) \stackrel{\Xi}{\longrightarrow} \bigodot^{3}(T M) \stackrel{X\lrcorner}{\longrightarrow} \bigodot^{2}(T M)
$$

for any 1-form $X_{a}$. The homomorphism $\Xi$ is constructed by forming and prolonging the metrisability equation (e.g. [7]) and is the natural first step [20] in searching for a metric in a given projective class. We should point out that these constructions are carried out having arbitrarily chosen a non-zero section $\epsilon^{a b c}$ of the line bundle $\Lambda^{3}(T M)$. However, since a different section only changes the scale of the various obstruction tensors at each point, whether they vanish or not is unaffected. In the main body of this article we shall restore precision by introducing projective weights, in effect a mechanism for keeping track of the scale of $\epsilon^{a b c}$.

Theorem 1.2 is established by a different route, which seemingly creates a proliferation of projectively invariant obstructions to metrisability. For example, if one considers tensors such as $T^{a b c d e f}$, taking values in $\bigodot^{6}(T M)$ and of degree 6 in the projective Weyl tensor, equivalently in the tensor $V^{a b}{ }_{c}$, then one finds an 8-dimensional space of obstructions in the 11-dimensional space of projective invariants of this type. Indeed, there is already a quadratic obstruction as follows. 
Theorem 1.3 In order that a projective structure [V] be metrisable, the invariant tensor

$$
Q_{a b}^{c}=\epsilon_{p q(a} V_{b)}^{p r} V_{r}^{q c}
$$

must vanish, whilst in general it does not.

And if one prefers a scalar obstruction, then there is one of degree 3 as follows.

Theorem 1.4 In order that a projective structure [ $\nabla]$ be metrisable, the invariant scalar

$$
S=\epsilon_{a b c} V^{a p}{ }_{q} V_{r}^{b q} V_{p}^{c r}
$$

must vanish, whilst in general it does not.

Besides proving these theorems, we shall provide a systematic way of creating many more invariants. Nevertheless, we shall show by examples, in Sects. 2.3 and 2.4, that this proliferation of invariants is insufficient to characterise the metrisable projective structures. In Sect. 3 we shall reformulate the problem for path geometries in terms of systems of two second order ODE for the unparametrised paths.

We would like to thank Katharina Neusser for pointing out the projectively invariant pairing (12) as a useful device in understanding the metrisability equation and also for helpful discussions concerning the form of the tensor $V^{a b}{ }_{c}$ for the Egorov and Newtonian structures in Sects. 2.3 and 2.4.

Finally, as detailed in Sect. 2.3, we would like to thank Vladimir Matveev for drawing our attention to an alternative proof [15] of the non-metrisability of the Egorov structure and also for his pertinent comments concerning the possible values of the degree of mobility of Riemannian and Lorentzian metrics in three dimensions.

\section{Projective structures and metrisability}

Let $M$ be a smooth manifold. Let us consider an equivalence class [ $\nabla]$ of torsion-free connections on $T M$, where to say that $\nabla$ and $\widehat{\nabla}$ belong to $[\nabla]$ is to say that there is a 1-form $\Upsilon_{a}$ such that

$$
\widehat{\nabla}_{a} X^{b}=\nabla_{a} X^{b}+\Upsilon_{a} X^{b}+\delta_{a}{ }^{b} \Upsilon_{c} X^{c}
$$

This is the condition for the geodesics sprays of $\nabla$ and $\widehat{\nabla}$ on $T M$ to have the same projection to $\mathbb{P}(T M)$. Therefore, it is exactly the condition that all connections in [ $\nabla]$ share the same unparametrised geodesics on $M$. In other words, the equivalence class $[\nabla]$ operationally defines what is a projective structure.

The curvature of a given connection $\nabla \in[\nabla]$ is defined by

$$
\left[\nabla_{a}, \nabla_{b}\right] X^{c}=R_{a b}{ }^{c} X^{d}
$$

and can be uniquely decomposed as

$$
R_{a b}{ }^{c}{ }_{d}=W_{a b}{ }^{c} d+\delta_{a}^{c} \mathrm{P}_{b d}-\delta_{b}^{c} \mathrm{P}_{a d}+\beta_{a b} \delta_{d}^{c}
$$


where $\beta_{a b}=-2 \mathrm{P}_{[a b]}$ and $W_{a b}{ }^{c} d$ is totally trace-free. Then $\mathrm{P}_{a b}$ is the projective Schouten tensor and $W_{a b}{ }_{d}$ is the projective Weyl tensor. If we change connection in the projective class using (5) then

$$
\widehat{\mathrm{P}}_{a b}=\mathrm{P}_{a b}-\nabla_{a} \Upsilon_{b}+\Upsilon_{a} \Upsilon_{b}, \quad \widehat{\beta}_{a b}=\beta_{a b}+2 \nabla_{[a} \Upsilon_{b]},
$$

whilst $W_{a b}{ }^{c} d$ remains unchanged.

We now specialise to the case when $M$ is 3-dimensional. Computing the effect of a change of connection (5) on a 3 -form $\eta_{a b c}$, we find that

$$
\begin{aligned}
\widehat{\nabla}_{a} \eta_{b c d} & =\nabla_{a} \eta_{b c d}-3 \Upsilon_{a} \eta_{b c d}-\Upsilon_{b} \eta_{a c d}-\Upsilon_{c} \eta_{b a d}-\Upsilon_{d} \eta_{b c a} \\
& =\nabla_{a} \eta_{b c d}-4 \Upsilon_{a} \eta_{b c d}
\end{aligned}
$$

and so if $M$ is oriented (as we shall suppose henceforth) and $\eta_{a b c}$ is chosen to be everywhere non-vanishing (we say that $\eta_{a b c}$ is a choice of scale), then we may specify $\Upsilon_{a}$ by requiring that $\nabla_{a} \eta_{b c d}-4 \Upsilon_{a} \eta_{b c d}=0$, thus obtaining a unique connection $\widehat{\nabla}$ in the projective class such that $\widehat{\nabla}_{a} \eta_{b c d}=0$. We shall refer to the connections obtained in this way as special. From (6), we find that

$$
\left[\nabla_{a}, \nabla_{b}\right] \eta_{c d e}=-4 \beta_{a b} \eta_{c d e}
$$

and conclude that $\beta_{a b}=0$ for special connections and hence that the Schouten tensor $\mathrm{P}_{a b}$ is symmetric. (If $M$ is not oriented, then we define a choice of scale to be a nowhere-vanishing section of $\left(\Lambda_{M}^{3}\right)^{2}$ instead, where $\Lambda_{M}^{3}$ is the bundle of 3-forms on $M$.) For any $w \in \mathbb{R}$, it is convenient to denote by $\mathcal{E}(w)$ the line bundle $\left(\Lambda_{M}^{3}\right)^{-w / 4}$, invariantly defined as the bundle whose fibre at $p \in M$ is the 1-dimensional vector space

$$
\left\{\phi: \Lambda_{+}^{3} T_{p}^{*} M \rightarrow \mathbb{R}: \phi(\lambda \omega)=\lambda^{w / 4} \phi(\omega) \text { for all } \lambda>0\right\}
$$

where $\Lambda_{+}^{3} T_{p}^{*} M$ denotes the 3 -forms at $p$ positive with respect to the orientation, and we shall refer to a section $\rho$ of $\mathcal{E}(w)$ as a projective density of weight $w$. There are canonical isomorphisms $\mathcal{E}(k)=\mathcal{E}(1)^{\otimes k}$ for $k \in \mathbb{Z}$. Also, by construction, there is an identification $\mathcal{E}(-4)=\Lambda_{M}^{3}$, which we shall write as $\rho \mapsto \rho \epsilon_{a b c}$ for $\rho$ of projective weight -4 . Equivalently, we have a canonical volume form $\epsilon_{a b c}$ of weight 4 , that is to say a canonical section of $\Lambda_{M}^{3}(4)$, the tensor product $\Lambda_{M}^{3} \otimes \mathcal{E}(4)$. Having done this, a scale may be alternatively specified as a nowhere-vanishing density $\sigma$ of projective weight 1 , so that $\eta_{a b c}=\sigma^{-4} \epsilon_{a b c}$ is the corresponding volume form. This is the viewpoint we shall adopt henceforth. In summary, we are working with special connections specified by a choice of projective density $\sigma$ of weight 1 . Choosing a different scale, say $\widehat{\sigma}=\Omega^{-1} \sigma$ for some nowhere-vanishing function $\Omega$, induces a projective change of connection (5) where $\Upsilon=\Omega^{-1} d \Omega$. In the presence of a scale $\sigma \in \Gamma(M, \mathcal{E}(1))$, we may view a projective density $\rho \in \Gamma(M, \mathcal{E}(w))$ as a smooth function, specifically $f=\rho / \sigma^{w}$, but if we change scale $\sigma \mapsto \widehat{\sigma}=\Omega^{-1} \sigma$, then this function changes according to $\widehat{f}=\Omega^{w} f$. Finally, for any scale $\sigma \in \Gamma(M, \mathcal{E}(1))$, the 
line bundles $\mathcal{E}(w)$ inherit connections characterised by $\nabla \sigma^{w}=0$ and then, if $\rho$ is a projective density of weight $w$, we see that

$$
\widehat{\nabla}_{a} \rho=\nabla_{a} \rho+w \Upsilon_{a} \rho
$$

and note that this is consistent with (7) and the identification $\Lambda_{M}^{3}=\mathcal{E}(-4)$. Otherwise said, the tautological 3-form $\epsilon_{a b c}$ of weight 4 is covariant constant $\left(\nabla_{a} \epsilon_{b c d}=0\right)$ for any special connection on $\Lambda_{M}^{3}(4)$. We shall denote by $\epsilon^{a b c}$ the induced canonical section of the dual bundle $\Lambda^{3}(T M)(-4)$ normalised so that

$$
\begin{aligned}
\epsilon_{a b c} \epsilon^{d e f}=\delta_{a}{ }^{d} \delta_{b}{ }^{e} \delta_{c}{ }^{f} & +\delta_{a}{ }^{e} \delta_{b}{ }^{f} \delta_{c}{ }^{d}+\delta_{a}{ }^{f} \delta_{b}{ }^{d} \delta_{c}{ }^{e} \\
& -\delta_{a}{ }^{e} \delta_{b}{ }^{d} \delta_{c}{ }^{f}-\delta_{a}{ }^{d} \delta_{b}{ }^{f} \delta_{c}{ }^{e}-\delta_{a}{ }^{f} \delta_{b}{ }^{e} \delta_{c}{ }^{d},
\end{aligned}
$$

equivalently that $\epsilon^{a b c} \epsilon_{a b c}=6$.

For those who find this discussion arcane, we should admit that a low-tech alternative is to regard formulæ containing $\epsilon^{a b c}$ as defined using an arbitrarily chosen nowhere-vanishing section $\epsilon^{a b c}$ of $\Lambda^{3}(T M)$ and that the projective weight simply keeps track of how these expressions change if $\epsilon^{a b c}$ is rescaled. Looking back at (1) now, we see that $V^{a b}{ }_{c}$ may be invariantly regarded as a tensor satisfying

$$
V_{c}^{a b}=V^{(a b)}, \quad V^{a b}{ }_{a}=0, \quad \text { and of projective weight }-4 .
$$

Similarly, the scalar invariant $S$ from Theorem 1.4 is a projective density of weight -8 .

Even more arcane, yet correspondingly even more useful, is to record both the symmetries of a tensor and its projective weight by decorating a suitable Dynkin diagram in the style of [1]. An explanation sufficient for our purposes is as follows. We shall denote by $\stackrel{k}{\times} \stackrel{l}{\longrightarrow} \quad m$ the bundle of totally trace-free tensors

$$
S^{\overbrace{a b \cdots c}^{m}} \underbrace{\text { contravariant indices }}_{l \text { covariant indices }} \text { of projective weight } k+2 l-m
$$

and symmetric in its covariant and contravariant indices. These constitute a complete list of the irreducible bundles on an oriented projective 3-manifold as a parabolic geometry in the sense of [3]. It is also useful to introduce the degree of such a bundle as

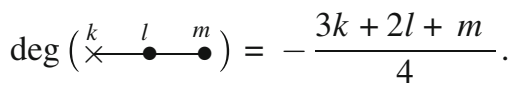


(It is the action of the grading element normalised as in [3].) The point about the degree is that it simply adds under tensor product, for example,

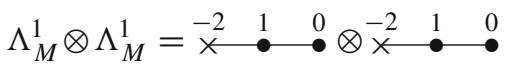

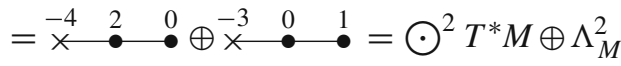

and changes sign when taking duals, for example,

$$
\operatorname{deg}(T M)=\operatorname{deg}\left(\begin{array}{lll}
\stackrel{1}{\longleftarrow} & 0 & 1 \\
\bullet & \bullet
\end{array}\right)=-1 \quad \text { whilst } \quad \operatorname{deg}\left(\Lambda_{M}^{1}\right)=\operatorname{deg}\left(\begin{array}{lll}
-2 & 1 & 0 \\
\longleftarrow & \bullet
\end{array}\right)=1 .
$$

Also notice that our previous discussion concerning projective weights and the tautologically defined tensors $\epsilon_{a b c}$ and $\epsilon^{a b c}$ is implicitly incorporated into this notation. For example, the identification $\Lambda_{M}^{2}=\stackrel{-3}{\longleftarrow} \bullet^{0} !$ in (9) is given by $\omega_{a b} \mapsto \epsilon^{a b c} \omega_{b c}$. Fundamental for this article is (1), giving $V^{a b}{ }_{c} \in \Gamma\left(M, \stackrel{-4}{\times} \quad \bullet^{-} \quad\right.$ ? $)$ of projective weight -4 . This irreducible tensor is every bit as good as the unweighted projectively invariant Weyl tensor $W_{a b}{ }^{c} d$, the inverse to (1) being given by $W_{a b}{ }^{c} d=\epsilon_{e a b} V^{e c} d / 2$.

\subsection{Metrisability}

As already remarked, a (pseudo-)Riemannian metric $g$ on $M$ gives rise to a projective structure $[\nabla]$, namely the one that contains the Levi-Civita connection of $g$. Hence we obtain a first order non-linear operator

$$
J^{1}\left(\bigodot^{2} \Lambda_{M}^{1}\right) \supset J^{1}\left(\bigodot_{\text {nd }}^{2} \Lambda_{M}^{1}\right) \stackrel{\sigma^{0}}{\longrightarrow} \operatorname{Pr}(M)
$$

which carries a metric to its associated projective structure, where $J^{1}\left(\bigodot_{\text {nd }}^{2} \Lambda_{M}^{1}\right)$ is a Zariski-open subbundle of the rank 24 first jet bundle (e.g. [24]), and the affine bundle $\operatorname{Pr}(M)$ of projective structures on $M$ is modelled on $\stackrel{-3}{\longleftarrow} \stackrel{1}{\longrightarrow}$, which has rank 15 .

Even taking into account that constant multiples of a given metric give rise to the same connection and hence the same projective structure, the dimensions indicate that $\sigma^{0}$ should be surjective and it is easy to check, using local coördinates, that this is, indeed, the case. Differentiating (10), however, gives rise to its first prolongation

$$
J^{2}\left(\bigodot_{\mathrm{nd}}^{2} \Lambda_{M}^{1}\right) \stackrel{\sigma^{1}}{\longrightarrow} J^{1}(\operatorname{Pr}(M))
$$

where the left hand side is now a Zariski-open subbundle of a vector bundle of rank 60 whilst the right hand side is an affine bundle modelled on $J^{1}\left(\begin{array}{ccc}-3 & 2 \\ \times & \bullet & 1\end{array}\right)$ a vector bundle also of rank 60. Taking scaling into account, it follows that $\sigma^{1}$ cannot be surjective. In other words, already at first order in the projective structure, we expect to 
see obstructions to metrisability. Theorem 1.1 shows that, indeed, there are obstructions at this order which are algebraic in components of the projective Weyl curvature. There is no restriction on the value of this curvature as the following lemma shows.

Lemma 2.1 Choose $n \geqslant 3$ and let $W_{a b}{ }^{c} d$ be any element of $\left(\mathbb{R}^{n}\right)^{*} \otimes\left(\mathbb{R}^{n}\right)^{*} \otimes \mathbb{R}^{n} \otimes$ $\left(\mathbb{R}^{n}\right)^{*}$ satisfying

$$
W_{a b}^{c} d=W_{[a b]}^{c} d, \quad W_{\left[a b^{c} d\right]}^{c}=0, \quad W_{a b}^{a} d=0 .
$$

Let $M$ be an n-dimensional manifold and let $p \in M$ be an arbitrarily chosen point. Then there is a torsion-free connection on TM whose projective Weyl curvature at $p$ is $W_{a b}{ }^{c} d$.

Proof The construction need only be local since connections can be patched together by a partition of unity. In local coördinates $\left(x^{1}, x^{2}, \ldots, x^{n}\right)$, the connection

$$
\nabla_{b} X^{c}=\frac{\partial X^{c}}{\partial x^{b}}+\frac{2}{3} x^{a} W_{a\left(b^{c} d\right)} X^{d}
$$

has projective Weyl curvature $W_{a b}{ }^{c} d$ at the origin.

In fact, the proof is also valid when $n=2$ but the statement is vacuous since only the zero tensor satisfies the symmetries (11). But for $n=3$ the space of the tensors satisfying (11) is 15-dimensional. This is clear from the alternative encoding of the Weyl curvature as the tensor $V^{a b}{ }_{c}$ in (1) satisfying $V^{a b}{ }_{c}=V^{(a b)}{ }_{c}$ and $V^{a b}{ }_{a}=0$. Alternatively, the dimension of any finite-dimensional irreducible tensor bundle can be computed from its highest weight and the Dynkin diagram notation established above is designed with this in mind. There are various algorithms and computer implementations thereof. For example

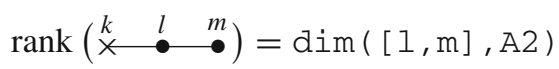

where the right hand side of this equation is an instruction written in $\mathrm{LiE}$ [16].

Henceforth, we shall use the terminology metric to mean (pseudo-)Riemannian metric. The signature plays no essential rôle in our considerations and can be discussed separately.

One can attack the metrisability problem directly, asking for a metric $g_{a b}$ such that its Levi-Civita connection be projectively equivalent to a given connection. Although the resulting partial differential equations on $g_{a b}$ are projectively invariant by construction, they are also non-linear. A surprising observation, essentially due to Liouville [17], is that there is a non-linear change of variables that turns this system into a linear one. For the convenience of the reader, we summarise the conclusions in three dimensions here and refer to $[7,19]$ for detail. 
Theorem 2.2 There is a projectively invariant linear differential operator

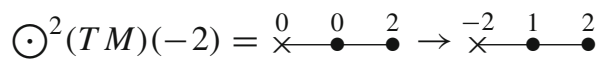

given by

$$
\sigma^{b c} \mapsto\left(\nabla_{a} \sigma^{b c}\right)_{\circ} \equiv \nabla_{a} \sigma^{b c}-\frac{1}{2} \delta_{a}{ }^{(b} \nabla_{d} \sigma^{c) d}
$$

called the metrisability operator and a projectively invariant differential pairing

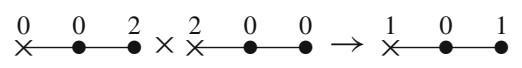

given by

$$
\left(\sigma^{a b}, \tau\right) \mapsto \sigma^{a b} \nabla_{b} \tau-\frac{1}{2} \tau \nabla_{b} \sigma^{a b}
$$

If $\sigma^{b c}$ is symmetric and of projective weight -2 , i.e. $\sigma^{b c} \in \Gamma\left(\begin{array}{lll}0 & 0 & 0 \\ \times & \bullet\end{array}\right)$, then

$$
\operatorname{det} \sigma \equiv \frac{1}{6} \sigma^{a d} \sigma^{b e} \sigma^{c f} \epsilon_{a b c} \epsilon_{d e f}
$$

is a projective density of weight 2 , i.e. $\operatorname{det} \sigma \in \Gamma\left(\begin{array}{ccc}2 & 0 & 0 \\ \times & \bullet\end{array}\right)$. If a tensor $\sigma^{b c} \in$ $\Gamma\left(\begin{array}{lll}0 & 0 & 2 \\ \times & \bullet & \bullet\end{array}\right)$ satisfies the projectively invariant metrisability equation

$$
\left(\nabla_{a} \sigma^{b c}\right)_{\circ}=0
$$

then the pairing with its determinant vanishes:

$$
\sigma^{a b} \nabla_{b}(\operatorname{det} \sigma)-\frac{1}{2}(\operatorname{det} \sigma) \nabla_{b} \sigma^{a b}=0
$$

Furthermore, wherever det $\sigma$ is non-zero, the weight zero tensor $g^{a b} \equiv(\operatorname{det} \sigma) \sigma^{a b}$ defines a metric whose Levi-Civita connection lies in the given projective class. Finally, up to sign, all metrics in a given projective class arise in this manner.

Proof As set forth in the statement of this theorem, these claims are straightforwardly verified from the definitions, the only further observation required being that (14) can be rewritten on $\{\operatorname{det} \sigma \neq 0\}$ as $\widehat{\nabla}\left(g^{a b}\right)=0$ where $\widehat{\nabla}_{a}$ is projectively equivalent to $\nabla_{a}$ according to (5) if we take $\Upsilon_{a}=-g_{a b}(\operatorname{det} \sigma) \nabla_{c} \sigma^{b c} / 4$, where $g_{a b}$ is the inverse to $g^{a b}$. (We have taken the opportunity here, following a suggestion of Katharina Neusser, to streamline the exposition in [7] by highlighting the rôle of (14).)

An informal summary of Theorem 2.2 is that the metrisability of a given projective structure is controlled by the projectively invariant metrisability equation (13), there being a 2-1 correspondence between non-degenerate solutions of this equation and positive metrics in the projective class (note that $\sigma^{a b}$ and $-\sigma^{a b}$ give rise to the same 
metric, that these metrics have positive determinant (we call them positive), and that conversely if $g^{a b}$ is such a metric, then

$$
\sigma^{a b} \equiv(\operatorname{det} g)^{-1 / 4} g^{a b}
$$

solves (13)).

Theorem 2.3 If $\sigma^{b c}$ solves the metrisability equation (13), then

$$
W_{a b}{ }^{c}{ }^{c} \sigma^{d e}+W_{a b}{ }^{d}{ }_{e} \sigma^{c e}+\frac{2}{3} \delta_{[a}^{c} W_{b]} e^{d}{ }_{f} \sigma^{e f}+\frac{2}{3} \delta_{[a}{ }^{d} W_{b]} e^{c}{ }^{c} \sigma^{e f}=0 .
$$

Proof If we write (13) as

$$
\nabla_{b} \sigma^{c d}=\delta_{b}^{c} \mu^{d}+\delta_{b}{ }^{d} \mu^{c}
$$

for some field $\mu^{a}$, then

$$
\left(\nabla_{a} \nabla_{b}-\nabla_{b} \nabla_{a}\right) \sigma^{c d}+2 \delta_{[a}^{c} \nabla_{b]} \mu^{d}+2 \delta_{[a}^{d} \nabla_{b]} \mu^{c}=0
$$

but from (6) using, without loss of generality, a special connection

$$
\left(\nabla_{a} \nabla_{b}-\nabla_{b} \nabla_{a}\right) \sigma^{c d}=W_{a b}^{c} e^{d e}+W_{a b}{ }^{d} e^{\sigma^{c e}}+2 \delta_{[a}{ }^{c} \mathrm{P}_{b] e} \sigma^{d e}+2 \delta_{[a}{ }^{d} \mathrm{P}_{b] e} \sigma^{c e}
$$

so it follows that

$$
W_{a b}{ }^{c} \sigma^{d e}+W_{a b}{ }^{d}{ }^{d} \sigma^{c e}+2 \delta_{[a}^{c} \Psi_{b]}^{d}+2 \delta_{[a}^{d} \Psi_{b]}^{c}=0
$$

where

$$
\Psi_{b}^{d}=\nabla_{b} \mu^{d}+\mathrm{P}_{b e} \sigma^{d e}
$$

Tracing (16) over ${ }_{b}^{c}$ yields

$$
W_{a b}{ }^{d} \sigma^{b e}-3 \Psi_{a}{ }^{d}+\delta_{a}{ }^{d} \Psi_{b}^{b}=0 .
$$

Finally, tracing this conclusion over $a^{d}$ shows that $\Psi_{b}{ }^{b}=0$ and substituting for $\Psi_{b}{ }^{d}$ back into (16) gives (15), as required.

We remark that it is usual to establish (15) by firstly prolonging the metrisability operator, as is done in [7], to obtain a projectively invariant connection on an auxiliary vector bundle whose curvature is then computed and found to include the left hand side of (15). It is already observed in [2] that (15) gives rise to non-trivial obstructions to metrisability. To express these obstructions in three dimensions, let us recall that $W_{a b}{ }^{c}{ }_{d}=\epsilon_{e a b} V^{e c} d 2$, which enables us to rewrite (15) as

$$
V^{(a b} \sigma^{c) d}=0
$$


or, in other words, that

$$
\Xi^{a b c}{ }_{d e} \sigma^{d e}=0, \quad \text { where } \Xi^{a b c}{ }_{d e}=V_{(d}^{(a b} \delta_{e)}{ }^{c)} .
$$

Taking projective weights into account, this defines an invariant homomorphism

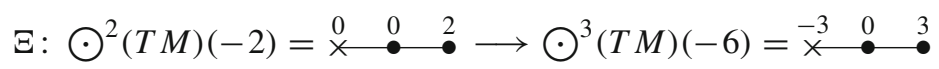

and Theorem 2.3 may be recast as follows.

Theorem 2.4 If $\sigma^{b c}$ solves the metrisability equation (13), then $\sigma^{b c}$ lies in the kernel of the endomorphism

$$
\Gamma\left(M, \bigodot^{2}(T M)(-2)\right) \ni \sigma^{d e} \mapsto X_{a} \Xi^{a b c} d e \sigma^{d e} \in \Gamma\left(M, \bigodot^{2}(T M)(-2)\right)
$$

for any projectively weighted 1 -form $X_{a}$ of weight 4 .

Proof of Theorem 1.1 This is an almost immediate corollary of Theorem 2.4. As already observed in the introduction, as $\bigodot^{2} T M(-2)$ is a vector bundle of rank 6 , the determinant of the endomorphism (18) has the form $X_{a} X_{b} X_{c} X_{d} X_{e} X_{f} T^{a b c d e f}$ for some projectively invariant

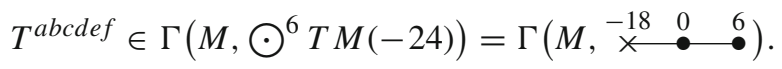

A priori this might always vanish but a suitable Weyl tensor $W_{a b}{ }^{c}$ is exhibited in [2, Sect. 8] with non-zero determinant (and this is realised by a projective structure in accordance with Lemma 2.1). It remains only to check that (2) gives a formula for $T$ but this is easily accomplished with the aid of computer algebra.

Since $V^{a b}{ }_{a}=0$, it follows that $\Xi^{a b c}{ }_{b c}=0$ whence the endomorphism $X_{a} \Xi^{a b c} d e$ is traceless for any $X_{a}$. If we set $\Xi_{X} b c d e \equiv X_{a} \Xi^{a b c}$, the Cayley-Hamilton Theorem for traceless $6 \times 6$ matrices now implies that the vanishing of $T^{a b c d e f}$ is equivalent to the vanishing of

$$
24 \operatorname{Tr}\left(\Xi_{X}^{6}\right)-18 \operatorname{Tr}\left(\Xi_{X}^{4}\right) \operatorname{Tr}\left(\Xi_{X}^{2}\right)-8\left(\operatorname{Tr}\left(\Xi_{X}^{3}\right)\right)^{2}+3\left(\operatorname{Tr}\left(\Xi_{X}^{2}\right)\right)^{3}
$$

which is a little easier to compute. Some further consequences of the vanishing of $T^{a b c d e f}$ have been analysed in $[4,20]$. Whilst a detailed analysis of the aforementioned prolonged system (as, for example, presented in [7, Thm. 3.1]) will surely lead to more obstructions, we do not pursue this here but, in the following section, opt for an alternative and more elementary approach. It leads to a plethora of invariant obstructions whose relation to the metrisability equation and its prolongation remains mysterious. 


\subsection{An elementary construction of obstructions}

In three dimensions, the curvature of a metric connection is entirely captured by the Ricci curvature. Specifically,

$$
R_{a b}{ }^{c}{ }_{d}=\delta_{a}^{c} R_{b d}-\delta_{b}^{c} R_{a d}-g_{a d} R_{b}^{c}+g_{b d} R_{a}^{c}-\frac{1}{2} R\left(\delta_{a}^{c} g_{b d}-\delta_{b}^{c} g_{a d}\right) .
$$

A metric connection is special and from (6) we see firstly that $\mathrm{P}_{a b}=R_{a b} / 2$ and hence that

$$
W_{a b}{ }^{c} d=R_{a b}{ }^{c} d-\delta_{a}{ }^{c} \mathrm{P}_{b d}+\delta_{b}{ }^{c} \mathrm{P}_{a d}=R_{a b}{ }^{c} d-\frac{1}{2} \delta_{a}^{c} R_{b d}+\frac{1}{2} \delta_{b}{ }^{c} R_{a d} .
$$

From (19) we deduce that for a metric connection in three dimensions,

$$
\begin{aligned}
W_{a b}{ }^{c} d=\frac{1}{2} \delta_{a}{ }^{c} R_{b d} & -\frac{1}{2} \delta_{b}{ }^{c} R_{a d}-g_{a d} R_{b}{ }^{c} \\
& +g_{b d} R_{a}{ }^{c}-\frac{1}{2} R \delta_{a}{ }^{c} g_{b d}+\frac{1}{2} R \delta_{b}{ }^{c} g_{a d}
\end{aligned}
$$

Therefore from its definition (1), we find that

$$
V^{a b}{ }_{c}=\epsilon^{d e a} W_{d e}{ }^{b} c=\epsilon^{b e a} R_{e c}-2 \epsilon_{c}{ }^{e a} R_{e}{ }^{b}+\epsilon_{c}{ }^{b a} R .
$$

Lemma 2.5 For a metric connection $V^{a b}{ }_{c}=2 R^{d(a} \epsilon^{b)} d c$.

Proof Though it might not appear so, the right hand of (20) is symmetric in $a b$ as may be verified by computing

$$
\begin{aligned}
\epsilon_{d a b}\left(\epsilon^{b e a} R_{e c}\right. & \left.-2 \epsilon_{c}^{e a} R_{e}{ }^{b}+\epsilon_{c}{ }^{b a} R\right) \\
& =2 \delta_{d}{ }^{e} R_{e c}-2\left(g_{b c} \delta_{d}^{e}-g_{d c} \delta_{b}{ }^{e}\right) R_{e}{ }^{b}-2 g_{d c} R=0 .
\end{aligned}
$$

Symmetrising term-by-term in (20) gives the required formula.

Remark 2.6 An effective replacement for Lemma 2.5 may alternatively be obtained from the metrisability equation (13) via the constraint (15), equivalently via (17). Specifically, given $V^{a b}{ }_{c} \in \Gamma(M, \underset{-4}{\times} \quad \bullet \quad ?)$ let us suppose that

$$
V^{(a b}{ }_{d} \sigma^{c) d}=0 \quad \text { for some non-degenerate } \sigma^{a b} \in \Gamma(M, \stackrel{0}{\times} \quad \stackrel{0}{\bullet} \quad \stackrel{2}{\bullet}),
$$

write $\sigma_{a b} \in \Gamma\left(M, \underset{-2}{\times} \stackrel{2}{\times} \quad \bullet\right.$ ) for the inverse of $\sigma^{a b}$, and consider the tensor

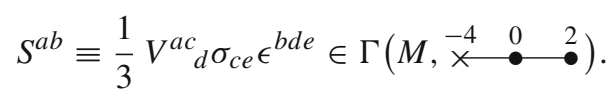


Then, one may readily verify that

$$
S^{a b}=S^{(a b)}, \quad S^{a b} \sigma_{a b}=0, \quad V^{a b}{ }_{c}=2 S^{d(a} \sigma^{b) e} \epsilon_{e d c}
$$

and that $S^{a b}$ is the unique tensor with these properties. The form of $V^{a b}{ }_{c}$ given here mimics the form given in Lemma 2.5 and is all that will be used in creating obstructions to metrisability. In the metrisable case, with metric $g^{a b}=(\operatorname{det} \sigma) \sigma^{a b}$, comparing these two forms shows that $(\operatorname{det} \sigma)^{-1} S_{a b}$ is the trace-free Ricci tensor. Although this route to $V^{a b}{ }_{c}=2 S^{d(a} \sigma^{b) e} \epsilon_{e d c}$ via the metrisability equation may seem rather convoluted, it was pointed out to us by the referee that similar manœuvres may be available in other prolonged systems possibly admitting no direct derivation.

At first glance, it may seem that Lemma 2.5 cannot be useful in restricting the possible Weyl curvature of a metrisable projective structure because the metric is already involved in the formula for $V^{a b}{ }_{c}$ especially via the tensor $\epsilon^{b} d c$. It turns out, however, that there are non-trivial projective covariants that necessarily vanish for $V^{a b}{ }_{c}$ of this special form no matter what metric and no matter what symmetric form $R^{d a}$ are chosen. The simplest example is $Q_{a b}{ }^{c}=\epsilon_{p q(a} V^{p r}{ }_{b)} V^{q c}$ from Theorem 1.3.

Proof of Theorem 1.3 Firstly, let us establish that $Q_{a b}{ }^{c}$ does not always vanish. A general method that is almost instantly effective with a computer is simply to compute all the coefficients of $Q_{a b}{ }^{c}$ as polynomials in, for example, the 15 variables

$$
\begin{aligned}
& V_{2}^{11}, \quad V_{3}^{11}, \quad V_{1}^{21}, \quad V_{2}^{21}, \quad V_{3}^{21}, \quad V_{1}^{22}, \quad V_{3}^{22}, \quad V_{1}^{31} \text {, } \\
& V_{2}^{31}, \quad V_{3}^{31}, \quad V_{1}^{32}, \quad V_{2}^{32}, \quad V_{3}^{32}, \quad V_{1}^{33}, \quad V_{2}^{33} \text {, }
\end{aligned}
$$

acting as coördinates on the space

$$
\left\{V^{a b}{ }_{c} \in \mathbb{R}^{3} \otimes \mathbb{R}^{3} \otimes\left(\mathbb{R}^{3}\right)^{*}: V^{a b}{ }_{c}=V^{(a b)}{ }_{c}, V^{a b}{ }_{a}=0\right\},
$$

any element of which can arise via (1) from the projective Weyl curvature of a suitable torsion-free connection in accordance with Lemma 2.1. Also, in this calculation, since there is only one totally skew 3-tensor up to scale, we may as well take

$$
\epsilon_{123}=\epsilon_{231}=\epsilon_{312}=1 \quad \text { and } \quad \epsilon_{213}=\epsilon_{132}=\epsilon_{321}=-1 \text {. }
$$

In this case, there is no real need for a computer to obtain, for example,

$$
Q_{33}{ }^{3}=V^{11}{ }_{3} V^{32}{ }_{1}-V^{31}{ }_{1} V^{21}{ }_{3}-V^{31}{ }_{2} V^{22}{ }_{3}+V^{21}{ }_{3} V^{32}
$$

and we are done. In fact, for low order invariants such as $Q_{a b}{ }^{c}$, glancing ahead to our more systematic investigation starting with Proposition 2.8, non-vanishing can also be seen without calculation as follows. The decomposition (22) proves the existence of a non-zero covariant $Q_{a b}{ }^{c}=Q_{(a b)}{ }^{c}$ and the only remaining issue is to find a formula for it. According to Weyl's first fundamental theorem of invariant theory [25] we are obliged to contract two copies of $V^{a b}{ }_{c}$ with (by counting the number of covariant and 
contravariant indices) one copy of $\epsilon_{a b c}$ and then take linear combinations. Bearing in mind the symmetries of $V^{a b}$, up to scale the only possibility for $Q_{a b}{ }^{c}$ is (4). This is especially clear using wiring diagrams as in [22]:

we know $\prod_{T}=0$ and $\zeta=0$ therefore $Q_{a b}{ }^{c}=\prod_{4}^{\square}$.

Now, we must show that $Q_{a b}{ }^{c}$ vanishes in the metrisable case. Well, if $V^{a b}{ }_{c}$ has the form given in Lemma 2.5 , then we compute

$$
\begin{aligned}
& \epsilon_{p q a} V_{b}^{p r} V_{r}^{q c}=\epsilon_{p q a}\left(R^{d p} \epsilon_{d b}^{r}+R^{d r} \epsilon_{d b}^{p}\right)\left(R^{e q} \epsilon_{e r}^{c}+R^{e c} \epsilon_{e r}^{q}\right) \\
&=\epsilon_{p q a} \epsilon_{d b}^{r} \epsilon_{e r}^{c} R^{d p} R^{e q}+\epsilon_{p q a} \epsilon_{d b}^{r} \epsilon_{e r}^{q} R^{d p} R^{e c} \\
& \quad+\epsilon_{p q a} \epsilon_{d b}^{p} \epsilon_{e r}^{c} R^{d r} R^{e q}+\epsilon_{p q a} \epsilon_{d b}^{p} \epsilon_{e r}^{q} R^{d r} R^{e c} \\
&=\epsilon_{p q a}\left(\delta_{d}^{c} g_{b e}-\delta_{b}{ }^{c} g_{d e}\right) R^{d p} R^{e q}+\epsilon_{p q a}\left(\delta_{d}^{q} g_{b e}-\delta_{b}{ }^{q} g_{d e}\right) R^{d p} R^{e c} \\
& \quad+\left(g_{q d} g_{a b}-g_{a d} g_{q b}\right) \epsilon_{e r}^{c} R^{d r} R^{e q}+\left(g_{q d} g_{a b}-g_{a d} g_{q b}\right) \epsilon_{e r}^{q} R^{d r} R^{e c} \\
&=\epsilon_{p q a} R^{c p} R_{b}{ }^{q}-\epsilon_{p q a} \delta_{b}{ }^{c} R^{d p} R_{d}^{q}+\epsilon_{p q a} R^{q p} R_{b}{ }^{c}-\epsilon_{p b a} R^{d p} R_{d}{ }^{c} \\
& \quad+g_{a b} \epsilon_{e r}^{c} R_{q}{ }^{r} R^{e q}-\epsilon_{e r}^{c} R_{a}{ }^{r} R^{e}{ }_{b}+g_{a b} \epsilon_{d e r} R^{d r} R^{e c}-\epsilon_{b e r} R_{a}{ }^{r} R^{e c} \\
&= \epsilon_{p q a} R^{c p} R_{b}{ }^{q}-\epsilon_{p b a} R^{d p} R_{d}{ }^{c}-\epsilon_{e r}^{c} R_{a}{ }^{r} R^{e}{ }_{b}-\epsilon_{b e r} R_{a}{ }^{r} R^{e c} \\
&= \epsilon_{p q a} R^{c p} R_{b}{ }^{q}+\epsilon_{a b p} R^{d p} R_{d}{ }^{c}+\epsilon_{r e}^{c} R_{a}{ }^{r} R_{b}{ }^{e}-\epsilon_{b p q} R_{a}{ }^{q} R^{c p},
\end{aligned}
$$

which is evidently skew in $a b$, as required.

Alternatively, we may compute in a preferred basis, the projective invariance ensuring that it does not matter what basis is chosen. In the Riemannian setting, for example, we may choose an orthonormal basis so that $g_{a b}$ is represented by the identity matrix and in addition choose $\epsilon_{a b c}$ to be the associated volume form. We may also diagonalise $R^{a b}$ and, optionally, remove its trace since $g^{a b}$ does not contribute to $V^{a b}{ }_{c}=2 R^{d(a} \epsilon^{b)} d c$. We leave the resulting verification to the reader. It is also straightforward to instruct a computer to work with these normalisations and this is our preferred method for analysing more complicated projective invariants. Finally, it is sufficient to work in the Riemannian setting:

Proposition 2.7 Working at a point (so that the following statement is purely algebraic) suppose a projective covariant constructed from $V^{a b}$ vanishes for all tensors of the form $V^{a b}{ }_{c}=2 R^{d(a} g^{b) e} \epsilon_{e d c}$ constructed from a fixed positive definite symmetric form $g^{a b}$, an associated volume form $\epsilon_{a b c}$, and an arbitrary trace-free symmetric form $R^{a b}$. Then the same covariant also vanishes for any non-degenerate $g^{a b}$.

The statement is clear by complexification.

In particular, when instructing a computer, it is sufficient to assume that $g^{a b}$ and $R^{a b}$ are simultaneously diagonalised: even though this might not be possible to arrange in the Lorentzian setting for example, as a statement of pure algebra it is densely true 
and this is good enough. In any case, we shall henceforth suppose that all metrics we encounter are positive definite.

Lemma 2.5 has a representation-theoretic interpretation as follows. Recall that on a 3-dimensional projective manifold, the tensor $V^{a b}$ is a section of $\varkappa^{-4} \stackrel{1}{\longleftarrow}$. This is an irreducible bundle but in the presence of a metric it decomposes according to the branching of the corresponding representation under

$$
\mathrm{GL}_{+}(3, \mathbb{R}) \supset \mathrm{SO}(3) \text {. }
$$

Specifically,

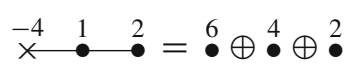

where $\stackrel{2 k}{\bullet}=\bigodot_{\circ}^{k} T M$ and $\circ$ denotes the trace-free tensors (and $\stackrel{1}{\bullet}, \stackrel{3}{\bullet}, \ldots$ denote spin bundles that need not concern us here). By Schur's Lemma, the homomorphisms ${ }^{4} \rightarrow$

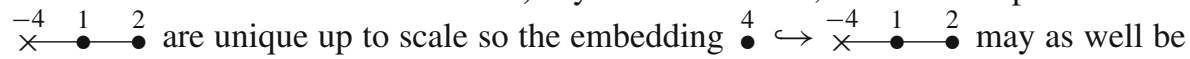
realised concretely by Lemma 2.5 . The other embeddings may as well be realised as

$$
\Gamma\left(M, \bigodot_{\circ}^{3} T M\right) \ni P^{a b c} \mapsto P_{c}^{a b} \quad \text { and } \quad \Gamma(M, T M) \ni L^{a} \mapsto L^{(a} \delta^{b)}{ }_{c}-2 g^{a b} L_{c}
$$

In order to construct potential obstructions by this approach, it is necessary firstly to construct projective covariants from the tensor $V^{a b}{ }_{c}$. It is straightforward to compute the locations and dimensions of such covariants. Quadratic covariants, for example, are limited by the following result.

Proposition 2.8 Up to scale, there are exactly five distinct quadratic covariants that may be constructed from $V^{a b}$, only one of which vanishes in the metrisable case.

Proof The usual theory of highest weights [11] allows us to decompose $\bigodot^{2}\left(\begin{array}{ccc}-4 & 1 & 2 \\ \hdashline & \bullet\end{array}\right)$ into its irreducible subbundles and this is easily implemented with a computer. For example, the program LiE [16] decomposes the symmetric tensor power of any irreducible representation of any simple algebra. In our case sym_tensor $(2,[1,2]$, A2 $)$

returns

$$
1 X[0,2]+1 X[1,3]+1 X[2,1]+1 X[2,4]+1 X[4,0] \text {, }
$$

which implies that

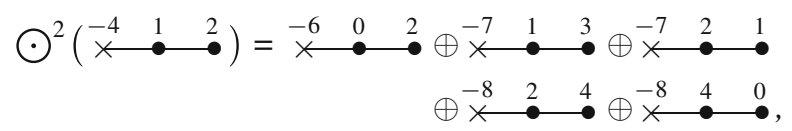

the numbers over the crossed nodes being controlled by the degree (8). This decomposition is exactly what we need to determine the location and multiplicity of quadratic covariants constructed from $V^{a b}$. The abstract theory does not give formulæ but, 
according to Weyl's first fundamental theorem of invariant theory [25], any such covariant can be expressed as a linear combination of contractions of $V^{a b}{ }_{c}$ itself with an appropriate multiplicity (in this case 2), together with the tautological form $\epsilon_{a b c}$ or its inverse $\epsilon^{a b c}$. In our case, we find

$$
\begin{aligned}
& A^{a b}=V_{q}^{a p} V_{p}^{b q}=V_{q}^{p(a} V_{p}^{b) q} \in \Gamma\left(\begin{array}{lll}
-6 & 0 & 2 \\
\longleftarrow & \bullet & \cdots
\end{array}\right), \\
& N^{a b c}{ }_{d}=5 V_{p}^{(a b} V_{d}^{c) p}-2 A^{(a b} \delta_{d}{ }^{c)} \in \Gamma\left(\begin{array}{ccc}
-7 & 1 & 3 \\
\times & \bullet
\end{array}\right) \text {, } \\
& Q_{a b}{ }^{c}=\epsilon_{p q(a} V_{b)}^{p r} V_{r}^{q c} \in \Gamma\left(\begin{array}{lll}
-7 & 2 & 1 \\
\times & \bullet
\end{array}\right) \text {, }
\end{aligned}
$$

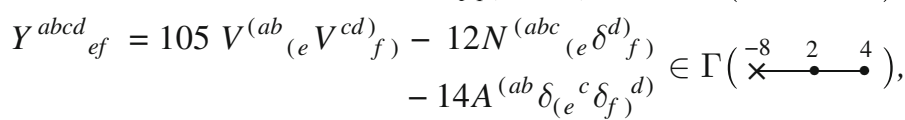

$$
\begin{aligned}
& Z_{a b c d}=\epsilon_{p r(a} V_{b}^{p q} V^{r s}{ }_{c} \epsilon_{d) q s} \in \Gamma\left(\begin{array}{lll}
-8 & 4 & 0 \\
\hdashline
\end{array}\right) \text {, }
\end{aligned}
$$

these formulæ being obtained by trial and error subject to the requirements only that the result be a non-vanishing tensor enjoying the specified symmetries (for then the formula is guaranteed by Schur's Lemma). It is then a matter of computation (best carried out with a computer) to check that only the invariant $Q_{a b}{ }^{c}$ vanishes when $V^{a b}{ }_{c}=2 R^{d(a} \epsilon^{b)} d c$.

Unfortunately, we only know how to prove Proposition 2.8 by direct calculation. Whilst we have no theoretical justification for why we might expect obstructions created in this way, the method of proof given above allows a systematic though computationally intensive method of finding many more as the following proofs show.

Proof of Theorem 1.4 In fact, noticing that

$$
S=\epsilon_{a b c} V^{a p}{ }_{q} V_{r}^{b q} V_{p}^{c r}=V^{a p} \epsilon_{a b c} V_{p}^{c r} V_{r}^{b q}=-V^{a b}{ }_{c} Q_{a b}{ }^{c},
$$

its vanishing in the metrisable case is immediate corollary of Theorem 1.3. A systematic approach to finding $S$, however, is to consider the decomposition

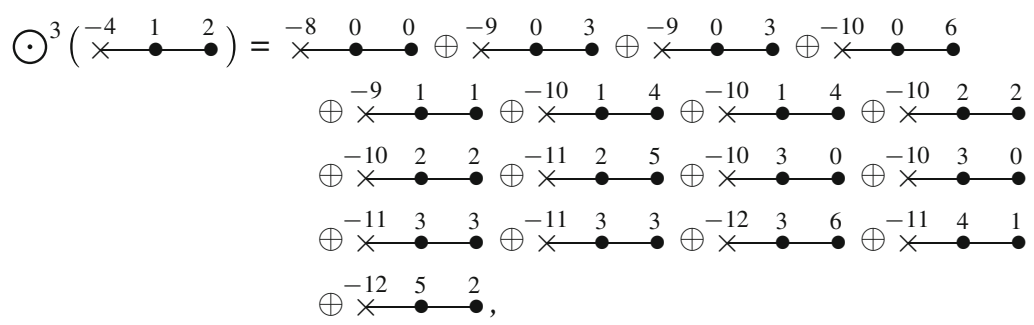

immediately obtained from LiE, write each of them as a linear combination of contractions, and then test each of these potential obstructions by substituting $V_{c}^{a b}=2 R^{d(a} \epsilon^{b)} d c$. This quickly leads to $S$ as stated in Theorem 1.4. Finally, 
the veracity of our claimed formula for $S$ may be instantly tested (with a computer) by simply calculating the result in our preferred variables (21), obtaining $S=6\left(V^{21}{ }_{1}\right)^{2} V^{31}{ }_{2}+3 V^{31}{ }_{1} V^{21}{ }_{1} V^{31}{ }_{3}+\cdots$, and observing that it is non-zero.

This reasoning leads to many other cubic covariants. One immediate difference to the decomposition (22), however, is that some subbundles occur with multiplicity. That

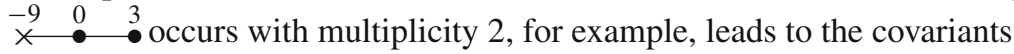

$$
B^{a b c}=\bigodot\left(V^{a p}{ }_{q} V_{r}^{b q} V_{p}^{c r}\right) \quad \text { and } \quad C^{a b c}=\bigodot\left(V^{a b}{ }_{p} V_{r}^{p q} V_{q}^{c r}\right)
$$

as stated in Theorem 1.1. Regarding the first conclusion of Theorem 1.2, it remains to check, by direct calculation, that $B^{a b c}$ and $C^{a b c}$ are linearly independent in general but that $C^{a b c}=2 B^{a b c}$ when $V^{a b}{ }_{c}$ is of the form $2 R^{d(a} \epsilon^{b)} d c$. In fact, the obstruction $C^{a b c}-2 B^{a b c}$ also arises from $Q_{a b}{ }^{c}$. Specifically,

$$
\begin{aligned}
2 & \epsilon^{p q a} Q_{p r}{ }^{b} V^{c r}{ }_{q} \\
& =2 \epsilon^{p q a} \epsilon_{d e}\left(p V^{d s}{ }_{r)} V^{e b}{ }_{s} V^{c r}{ }_{q}\right. \\
& =\epsilon^{p q a} \epsilon_{d e p} V^{d s}{ }_{r} V^{e b}{ }_{s} V^{c r}{ }_{q}+\epsilon^{p q a} \epsilon_{d e r} V^{d s}{ }_{p} V^{e b}{ }_{s} V^{c r}{ }_{q} \\
& =\left(\delta_{d}{ }^{q} \delta_{e}{ }^{a}-\delta_{d}{ }^{a} \delta_{e}{ }^{q}\right) V^{d s}{ }_{r} V^{e b}{ }_{s} V^{c r}{ }_{q}+\epsilon^{p q a} \epsilon_{d e r} V^{d s}{ }_{p} V^{e b}{ }_{s} V^{c r}{ }_{q} \\
& =V^{q s}{ }_{r} V^{a b}{ }_{s} V^{c r}{ }_{q}-V^{a s}{ }_{r} V^{q b}{ }_{s} V^{c r}{ }_{q}+\left(\delta_{d}{ }^{p} \delta_{e}{ }^{q} \delta_{r}{ }^{a}+\cdots\right) V^{d s}{ }_{p} V^{e b}{ }_{s} V^{c r}{ }_{q} \\
& =2 V^{a b}{ }_{p} V^{p q}{ }_{r} V^{c r}{ }_{q}-V^{a c}{ }_{p} V^{p q}{ }_{r} V^{b r}{ }_{q}-2 V^{a p}{ }_{q} V^{c q}{ }_{r} V^{b r}{ }_{p}
\end{aligned}
$$

and so

$$
2 \epsilon^{p q(a} Q_{p r}^{b} V_{q}^{c) r}=C^{a b c}-2 B^{a b c} .
$$

Proof of Theorem 1.2 We have just shown that $C^{a b c}-2 B^{a b c}=0$ in the metric case, either by direct computation or as a consequence of the vanishing of the quadratic covariant $Q_{a b}{ }^{c}$ from Theorem 1.3. Generally, it is non-zero. The remaining claims in Theorem 1.2 may be straightforwardly checked by direct computation (with a computer).

It is unclear whether all obstructions in Theorem 1.2 may be written in terms of $Q_{a b}{ }^{c}$. More generally, it is straightforward to generate many more invariant obstructions all of which may yet arise from the basic obstruction $Q_{a b}{ }^{c}$. We leave this question for a future investigation and content ourselves with the following complete determination of the sextic obstructions taking values in $\stackrel{-18}{\longleftarrow} \quad 6^{6}$ (as does $T^{\text {abcddef }}$ from Theorem 1.1).

Theorem 2.9 There is an 11-dimensional space of covariants of $V^{a b}{ }_{c}$ of degree 6 taking values in ${ }^{-18} \stackrel{0}{\bullet}$, an 8-dimensional subspace of which vanishes in the metrisable case.

Proof The first statement is that $\longrightarrow$ - 6 occurs with multiplicity 11 in $\odot^{6}\left(\begin{array}{ccc}-4 & 1 & 2 \\ \hdashline & \bullet & \bullet\end{array}\right)$ and, since $\operatorname{deg}\left(\left(\begin{array}{ccc}-18 & 0 & 6 \\ \longleftrightarrow & \bullet & \bullet\end{array}\right)=12=6 \times \operatorname{deg}\left(\begin{array}{lll}-4 & 1 & 2 \\ \longleftrightarrow & \bullet\end{array}\right)\right.$ from (8), 


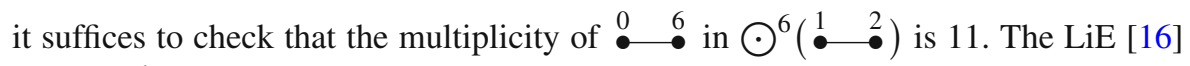
command

$$
\text { sym_tensor }(4,[1,2], \mathrm{A} 2) \mid[0,4]
$$

confirms this. Finding a basis for this space is then a matter of trial and error. For this purpose, rather than using indices, it is easier to write covariants using wiring diagrams as was done in the $19^{\text {th }}$ century [6] (see also [22]). Thus, the covariants $A^{a b}, B^{a b c}$, and $C^{a b c}$ from Theorem 1.1 are written as

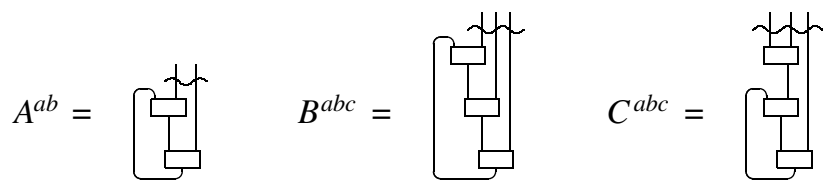

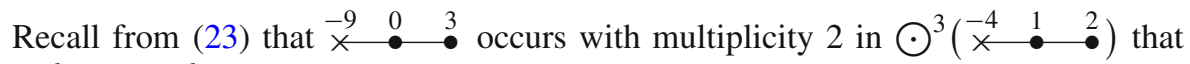
$B^{a b c}$ and $C^{a b c}$ span the covariants of this type. At quartic level,

$$
\text { sym_tensor }(6,[1,2], \text { A2 }) \mid[0,6]
$$

returns 4 and we already have $A^{(a b} A^{c d)}$ so we are looking for three more linearly independent covariants. The following suffice:

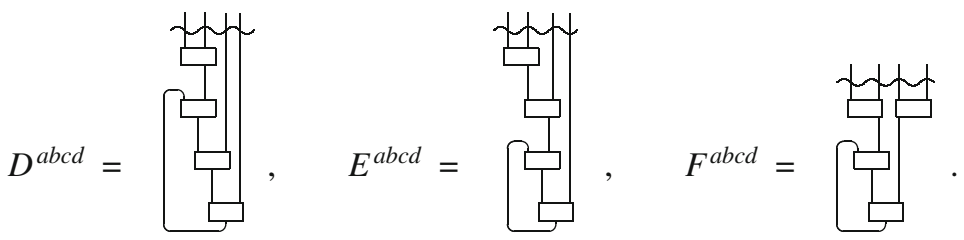

Note that one might naïvely also expect to encounter the covariant

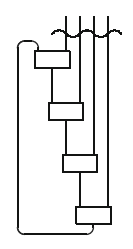

and in higher dimensions this would, indeed, be an independent covariant. In three dimensions, however, it turns out that this is $A^{(a b} A^{c d)} / 2$. In principle, such relations are covered by Weyl's second fundamental theorem of invariant theory [25], which says that they all arise by 'skewing over too many indices,' in this case 4 (the CayleyHamilton Theorem being a familiar example of such dimension-dependent relations). In practise, however, it is best to write down all potential covariants and allow a computer to sort out the relations. 
For completeness, we now move on to quintic invariants. Since

$$
\text { sym_tensor }(5,[1,2], A 2) \mid[0,5]
$$

returns 5 we need three more invariants to complement $A^{(a b} B^{c d e)}$ and $A^{(a b} C^{c d e)}$. It turns out that

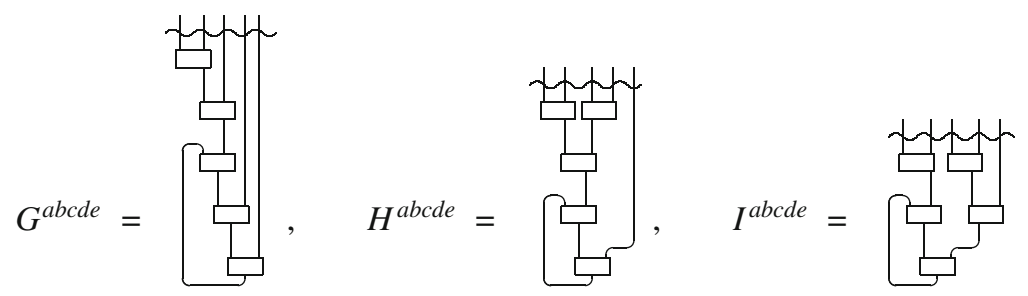

will suffice (amongst the seven possible quintic wiring diagrams beyond $A^{(a b} B^{c d e)}$ and $\left.A^{(a b} C^{c d e)}\right)$.

Finally we come to identify the sextic invariants and already we have

$$
\begin{aligned}
& A^{(a b} A^{c d} A^{e f)}, \quad B^{(a b c} B^{d e f)}, \quad C^{(a b c} C^{d e f)}, \\
& B^{(a b c} C^{d e f)}, \quad A^{(a b} D^{c d e f)}, \quad A^{(a b} E^{c d e f)}, \quad A^{(a b} F^{c d e f)} .
\end{aligned}
$$

It remains to find four more and it turns out that

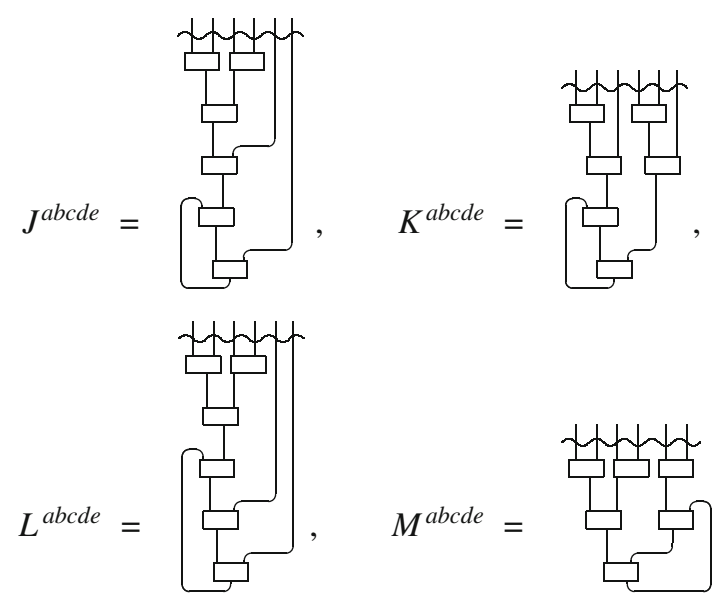

will suffice. It is then a matter of computation (with a computer) to check that, in addition to the five obstructions listed in Theorem 1.2, there are two more, namely

$$
E^{a b c d}-F^{a b c d} \text { and } J^{a b c d e f}-M^{a b c d e f},
$$

but that there are no more relations amongst $A, B, C, D, E, F, J, K, L$. Therefore, amongst the 11-dimensional space of covariants of degree 6 , the subspace comprising those that vanish in the metrisable case is 8-dimensional and is spanned by 


$$
\begin{array}{llll}
J-M, & J-2 L, & 3 J-2 C \odot C, & J-4 K+4 A \odot D, \\
A \odot E-A \odot F, & A \odot F-2 A \odot D, & B \odot C-2 B \odot B, & C \odot C-2 B \odot C,
\end{array}
$$

for example.

\subsection{Egorov's projective structure}

A projective symmetry is a vector field whose local flow maps unparametrised geodesics to unparametrised geodesics. Such symmetries form a Lie algebra $\mathfrak{g}$ under Lie bracket and, for connected 3-dimensional projective structures, we have dim $\mathfrak{g} \leqslant 15$ with equality if and only if $\mathfrak{g}=\mathfrak{s l}(4, \mathbb{R})$ in which case the structure is projectively flat, equivalently $V^{a b}{ }_{c} \equiv 0$.

The submaximal dimension for simply-connected 3-dimensional projective structures is 8 . The corresponding projective structure was given by Egorov [8] and can be represented in local coördinates $\left(x^{1}, x^{2}, x^{3}\right)$ as

$$
\nabla_{2} X^{1}=\partial_{2} X^{1}+x^{2} X^{3}, \quad \nabla_{3} X^{1}=\partial_{3} X^{1}+x^{2} X^{2}, \text { else } \nabla_{a} X^{c}=\partial_{a} X^{c},
$$

where $\partial_{a} \equiv \partial / \partial x^{a}$. For this connection, the only non-zero components of curvature are

$$
R_{23}{ }^{1}=-1, \quad R_{32}{ }^{1}{ }_{2}=1
$$

so $R_{a b}{ }^{c} d$ is already trace-free. Therefore $W_{a b}{ }^{c} d=R_{a b}{ }^{c}$. Hence

$$
V_{2}^{11}=-2 \quad \text { with all other components zero. }
$$

Immediately (17) implies $\sigma^{12}=\sigma^{22}=\sigma^{23}=0$ and then we can solve (13) explicitly:

$$
\sigma^{a b}=\left[\begin{array}{ccc}
A-B\left(x^{2}\right)^{2}+C\left(x^{2}\right)^{4} & 0 & B-2 C\left(x^{2}\right)^{2} \\
0 & 0 & 0 \\
B-2 C\left(x^{2}\right)^{2} & 0 & 4 C
\end{array}\right]
$$

for arbitrary constants $A, B, C$. Since all solutions are degenerate, we have shown:

Proposition 2.10 The Egorov projective structure is not metrisable.

Another proof As soon as the dimension of the solution space to (13) reaches 3, the projective structure cannot be metrisable unless it is projectively flat (it is easy to check that (26) solves (13) and that the projective Weyl curvature is non-vanishing without knowing that (26) is the general solution). In general, the degree of mobility of a metric is the dimension of the solution space of (13) for the associated projective structure and in three dimensions it can only be 1, 2, or 10 (as shown in [14] in the Riemannian case and [12] in the Lorentzian case (see [13,23] in the Riemannian setting and [9] in the Lorentzian setting for a detailed analysis concerning possible values of the degree of mobility in all dimensions (in [9], a detailed analysis is conducted 
under the assumption that there are at least two metrics in the projective class whose corresponding Levi-Civita connections are different but if this is not the case, then this is a sufficient imposition on the projective Weyl curvature that it must vanish))). Alternatively, Kruglikov and Matveev [15] consider the dimension of the space of local projective symmetries to conclude that the Egorov structure is not metrisable. Specifically, in three dimensions they show that the dimension of this space is bounded by 5 if there is a Riemannian metric inducing the projective structure and 6 if there is a Lorentzian metric inducing the projective structure (whereas, as noted above, the local projective symmetries are 8-dimensional for the Egorov structure).

Yet another proof We consider the projective Weyl curvature of the Egorov structure in comparison with Lemma 2.5. To make the comparison, let us use the metric to lower indices:

$$
V_{a b c}=2 R_{(a}^{d} \epsilon_{b) d c}, \quad \text { which implies that } V_{(a b c)}=0 .
$$

As a consequence of (25), however, $V^{a b}{ }_{c}$ is simple, i.e. there are non-zero $X^{a}$ and $Y_{a}$ such that $V^{a b}{ }_{c}=X^{a} X^{b} Y_{c}$. Therefore we have $V_{(a b c)}=X_{(a} X_{b} Y_{c)} \neq 0$, in conflict with (27).

Thus, we have yet another proof, this one just from the Weyl curvature, that the Egorov projective structure is not metrisable. On the other hand, for $V^{a b}{ }_{c}$ as in (25), projecting $V \odot V \odot \cdots \odot V$ into any component of

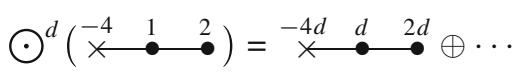

other than the first evidently gives zero. Thus, we see that the Weyl curvature is sufficiently special that the structure cannot be metrisable but that this situation cannot be detected by the vanishing of any projective covariant.

Finally, we remark that, although the Weyl tensor $V^{a b}{ }_{c}$ for the Egorov structure does not have the form required by Lemma 2.5 , it can still be written as

$$
V^{a b}{ }_{c}=2 R^{d(a} g^{b) e} \epsilon_{e d c}, \quad \text { where } R^{a b}=\left[\begin{array}{lll}
0 & 0 & 1 \\
0 & 0 & 0 \\
1 & 0 & 0
\end{array}\right] \text { and } g^{a b}=\left[\begin{array}{lll}
1 & 0 & 0 \\
0 & 0 & 0 \\
0 & 0 & 0
\end{array}\right] \text {, }
$$

which is of the required form save for $g^{a b}$ being degenerate.

\subsection{Newtonian projective structures}

In this section we construct some more non-metrisable projective structures notwithstanding that, again, all our obstructions vanish. Indeed, any obstruction that depends continuously on the projective structure will vanish for these Newtonian projective structures because they are created as limits of metrisable structures as follows. In local coördinates $\left(x^{1}, x^{2}, x^{3}\right)$, consider the metric

$$
\epsilon\left(\left(d x^{1}\right)^{2}+\left(d x^{2}\right)^{2}\right)+\exp \left(\epsilon f\left(x^{1}, x^{2}\right)\right)\left(d x^{3}\right)^{2}
$$


where $\epsilon \neq 0$ is constant and $f\left(x^{1}, x^{2}\right)$ is an arbitrary smooth function. The corresponding projective structures are metrisable by definition but if we let $\epsilon \rightarrow 0$, then these metric connections have a perfectly good limit, namely

$$
\begin{aligned}
& \nabla_{3} X^{1}=\partial_{3} X^{1}-\frac{1}{2}\left(\partial_{1} f\right) X^{3}, \quad \nabla_{3} X^{2}=\partial_{3} X^{2}-\frac{1}{2}\left(\partial_{2} f\right) X^{3}, \\
& \text { else } \quad \nabla_{a} X^{c}=\partial_{a} X^{c}
\end{aligned}
$$

(whose geodesic equations are Newton's equations for a particle in the $\left(x^{1}, x^{2}\right)$-plane moving under the influence of the potential $f\left(x^{1}, x^{2}\right)$ with $x^{3}=$ 'time') whereas

Proposition 2.11 Unless projectively flat, the Newtonian projective structures (28) are not metrisable.

Proof Firstly, we compute the curvature of (28)

$$
\begin{aligned}
& R_{13}{ }_{3}=-\frac{1}{2}\left(\partial_{1}\right)^{2} f, \quad R_{13}^{2}{ }_{3}=-\frac{1}{2} \partial_{1} \partial_{2} f, \quad R_{23} 1_{3}=-\frac{1}{2} \partial_{1} \partial_{2} f, \\
& R_{23}{ }^{2}{ }_{3}=-\frac{1}{2}\left(\partial_{2}\right)^{2} f, \quad R_{31}{ }^{1}{ }_{3}=\frac{1}{2}\left(\partial_{1}\right)^{2} f, \quad R_{31}{ }^{2}{ }_{3}=\frac{1}{2} \partial_{1} \partial_{2} f, \\
& R_{32}{ }^{1}{ }_{3}=\frac{1}{2} \partial_{1} \partial_{2} f, \quad R_{32}{ }^{2}{ }_{3}=\frac{1}{2}\left(\partial_{2}\right)^{2} f
\end{aligned}
$$

to discover that $\mathrm{P}_{33}=-\left(\left(\partial_{1}\right)^{2}+\left(\partial_{2}\right)^{2}\right) f / 4$ with all other components zero and hence that

$$
V^{a b}{ }_{c}=\epsilon^{d e a} W_{d e}{ }^{b}=\epsilon^{d e a} R_{d e}{ }^{b}{ }_{c}-2 \epsilon^{b e a} \mathrm{P}_{e c}
$$

is given by

$$
V_{3}^{a b}=\left[\begin{array}{ccc}
-\partial_{1} \partial_{2} f & \left(\left(\partial_{1}\right)^{2}-\left(\partial_{2}\right)^{2}\right) f / 2 & 0 \\
\left(\left(\partial_{1}\right)^{2}-\left(\partial_{2}\right)^{2}\right) f / 2 & \partial_{1} \partial_{2} f & 0 \\
0 & 0 & 0
\end{array}\right]
$$

with all other components zero. If $f\left(x^{1}, x^{2}\right)=\alpha x^{1}+\beta x^{2}+\gamma$, then $V^{a b}{ }_{c}$ vanishes and the structure is projectively flat. Otherwise the constraint (17) implies that $\sigma^{c 3}=0$ for all $c$. Already, all solutions to (13) are degenerate so the structure is not metrisable. In fact, one can go on to check that

$$
\sigma^{a b}=\left[\begin{array}{ccc}
A & B & 0 \\
B & C & 0 \\
0 & 0 & 0
\end{array}\right]
$$

is the general solution of (13) for arbitrary constants $A, B, C$. As for the Egorov example, this 3-dimensional space of solutions also precludes metrisability. 
Yet another proof As for the Egorov example, the form of the projective Weyl curvature (29) conflicts with Lemma 2.5 to provide yet another proof. This time $V^{a b}{ }_{c}$ has the form $X^{a b} Y_{c}$ for non-zero $X^{a b}$ and $Y_{a}$ (with $X^{a b}$ symmetric but never simple). Lowering the indices with the purported metric gives $V_{(a b c)}=X_{(a b} Y_{c)} \neq 0$, contrary to $(27)$.

Finally, we remark that, although the Weyl tensor $V^{a b}{ }_{c}$ for the Newtonian structure does not have the form required by Lemma 2.5 , it can still be written as

$$
\begin{aligned}
& V^{a b}{ }_{c}=2 R^{d(a} g^{b) e} \epsilon_{e d c}, \\
& \text { where } R^{a b}=-\frac{1}{2}\left[\begin{array}{ccc}
\partial_{1} \partial_{1} f & \partial_{1} \partial_{2} f & 0 \\
\partial_{2} \partial_{1} f & \partial_{2} \partial_{2} f & 0 \\
0 & 0 & 0
\end{array}\right] \text { and } g^{a b}=\left[\begin{array}{lll}
1 & 0 & 0 \\
0 & 1 & 0 \\
0 & 0 & 0
\end{array}\right] \text {, }
\end{aligned}
$$

which is of the required form save for $g^{a b}$ being degenerate.

\subsection{A Weyl metrisable but not metrisable projective structure}

Recall that a Weyl structure on $M$ consists of a conformal structure $[g]$ together with a torsion-free connection $D$ that is compatible with the conformal structure in a sense that

$$
D g=\omega \otimes g
$$

for $g \in[g]$ and some 1 -form $\omega$, this compatibility condition being invariant under the transformation

$$
g \mapsto \widehat{g} \equiv \Theta^{2} g, \quad \omega \mapsto \omega+2 d(\ln \Theta),
$$

where $\Theta$ is a non-zero function on $M$. For any metric $g$ in the conformal class, the 1-form $\omega$ determines the connection $D$.

Consider a Lorentizian Weyl structure on the three-dimensional Heisenberg group [21]

$$
g=\left(d x^{1}\right)^{2}-\left(d x^{2}\right)^{2}+\left(d x^{3}-x^{1} d x^{2}\right)^{2}, \quad \omega=2\left(d x^{3}-x^{1} d x^{2}\right) .
$$

This Weyl structure is Einstein-Weyl: the symmetrised Ricci tensor of $D$ is proportional to $g$. Let $[D]$ be the projective structure defined by $D$.

We find that the obstruction $Q_{a b}{ }^{c}$ from Theorem 1.3 does not vanish. For example, $Q_{22}{ }^{1}=x^{1}$. The determinant $T$ from Theorem 1.1 also does not vanish. A convenient way to present $T$ is to regard it as a ternary sextic. Setting $X_{a}=(X, Y, Z)$ we find

$$
X_{a} X_{b} X_{c} X_{d} X_{e} X_{f} T^{a b c d e f}=Z^{2}\left(X+Y+Z x^{1}\right)^{2}\left(X-Y-Z x^{1}\right)^{2}
$$

up to a non-zero multiplicative constant.

From either of these obstructions, we therefore conclude that the projective structure $[D]$ is not metrisable. It is nevertheless Weyl metrisable by construction. In dimension 
two all projective structures are locally Weyl metrisable [18]. We expect this not to be the case in dimension three, where, up to diffeomorphism, a general real-analytic projective structure depends on twelve arbitrary functions of three variables, but a Weyl structure only depends on five such functions. Characterising projective connections that are Weyl metrisable is an interesting open problem, which we do not pursue here. In general, we also do not know which Weyl metrisable structures are genuinely metrisable. If the Einstein-Weyl equations hold, however, then we have a satisfactory answer as follows.

\subsection{Einstein-Weyl projective structures}

Consider a 3-dimensional Weyl structure $(D,[g])$ as outlined at the beginning of the previous section. In general, the Ricci tensor of $D$ contains both symmetric and skew parts, the latter being proportional to $\nabla_{[a} \omega_{b]}$. The 2-form $F_{a b} \equiv \nabla_{[a} \omega_{b]}$ is an invariant of the Weyl structure, often called the Faraday form. The Weyl structure is called Einstein-Weyl if

$$
\Phi_{a b}=0,
$$

where $\Phi_{a b}$ is the symmetrised trace-free part of the Ricci tensor of $D$ (noting that removing the trace of a symmetric tensor depends only on the conformal class $[g]$ ).

Theorem 2.12 Let $(D,[g])$ be an Einstein-Weyl structure in dimension 3, and let $[D]$ be the projective structure defined by $D$. Then $[D]$ is metrisable if and only if its Faraday form $F_{a b}$ vanishes.

Proof Let $V^{a b}{ }_{c}$ be as usual (1). A straightforward but cumbersome calculation yields

$$
V_{c}^{a b}=2 \Phi^{d(a} \epsilon^{b)} d c-\frac{1}{2} \delta_{c}^{(a} f^{b)}+g^{a b} f_{c}
$$

where $f^{a}$ is the vector field defined by

$$
F_{a b}=\epsilon_{a b c} f^{c}
$$

and indices are lowered and raised by any representative metric $g_{a b}$ from the conformal class and its inverse $g^{a b}$. An even more cumbersome calculation gives

$$
Q_{a b}{ }^{c}=\Phi_{a b} f^{c}+2 \Phi_{(a}^{c} f_{b)}-2 \delta^{c}{ }_{(a} \Phi_{b)}^{d} f_{d}+2 g_{a b} \Phi^{c d} f_{d}+f_{(a} \epsilon_{b) d}{ }^{c} f^{d},
$$

where $Q_{a b}{ }^{c}$ is our usual quadratic obstruction (4) to metrisability. If the Einstein-Weyl equations (31) hold then $Q_{a b}{ }^{c}$ vanishes if and only if $f^{a}=0$, which happens if and only if $\omega$ is locally a gradient. But in this case the Weyl connection is the Levi-Civita connection of a conformally rescaled metric $\widehat{g}$, so it is metrisable (as it is metric).

In fact, if the Faraday form vanishes, and locally we choose a metric connection in the projective class according to (30), then the Einstein-Weyl equations (31) revert to 
the Einstein equations. Since we are in three dimensions, the Einstein equations imply that the metric is constant curvature. Therefore, the only metrisable Einstein-Weyl structures in three dimensions are projectively flat.

\section{Path geometries and systems of ODEs}

A convenient way to exhibit examples of projective structures on $U^{\text {open }} \subseteq \mathbb{R}^{3}$ is to use an equivalent definition of a path geometry in three dimensions as an equivalence class of systems of two second order ordinary differential equations [5]

$$
y^{\prime \prime}=F\left(x, y, z, y^{\prime}, z^{\prime}\right), \quad z^{\prime \prime}=G\left(x, y, z, y^{\prime}, z^{\prime}\right)
$$

where two systems are regarded as equivalent if they can be mapped into each other by a change of dependent and independent variables

$$
(x, y, z) \mapsto(\bar{x}(x, y, z), \bar{y}(x, y, z), \bar{z}(x, y, z)) .
$$

An integral curve of (32) is, for sufficiently regular functions $F, G$, specified uniquely by a point and a direction in $U$.

It is relatively straightforward to characterise 2 nd order systems (32) that give rise to projective path geometries [4,10]: set $y^{i}=(y, z), p^{i}=\left(y^{\prime}, z^{\prime}\right)$ and $F^{i}=$ $(F, G)$, where the indices $i, j, k, \ldots$ take values 2,3 . The necessary and sufficient conditions for the integral curves of (32) to be unparametrised geodesics of a torsionfree connection on $T U$ are [10]

$$
S_{(j k l)}^{i}=0, \quad \text { where } \quad S_{j k l}^{i}=\frac{\partial^{3} F^{i}}{\partial p^{j} \partial p^{k} \partial p^{l}}-\frac{3}{4} \frac{\partial^{3} F^{m}}{\partial p^{m} \partial p^{j} \partial p^{k}} \delta_{l}^{i} .
$$

To establish this result it is enough to consider the geodesic equations for a given $\nabla$, and eliminate the affine parameter $s$ between the three equations

$$
\frac{d^{2} x^{a}}{d s^{2}}+\Gamma_{b c}{ }^{a} \frac{d x^{b}}{d s} \frac{d x^{c}}{d s}=0
$$

where $x^{a}=(x, y, z)$. This yields (32), with

$$
F^{i}=A_{j k} p^{i} p^{j} p^{k}+B_{j k}^{i} p^{j} p^{k}+C_{j}^{i} p^{j}+D^{i}
$$

where

$$
\begin{array}{ll}
A_{i j}=\Gamma_{i j}{ }^{1}, & B^{i}{ }_{j k}=2 \Gamma_{1\left(j^{1} \delta_{k)}{ }^{i}-\Gamma_{j k}{ }^{i},\right.} \\
C^{i}{ }_{j}=\Gamma_{11}{ }^{1} \delta_{j}{ }^{i}-2 \Gamma_{1 j}{ }^{i}, & D^{i}=-\Gamma_{11}{ }^{i} .
\end{array}
$$

Note that the expressions for $A, B, C, D$ are invariant under (5). Conversely, imposing (33) on system (32) yields (34) as in [4]. For example the Egorov projective structure (24) corresponds to a system 


$$
y^{\prime \prime}=2 y\left(y^{\prime}\right)^{2} z^{\prime}, \quad z^{\prime \prime}=2 y y^{\prime}\left(z^{\prime}\right)^{2} .
$$

Expressing any of the projective invariants in this article, such as (3), in terms of $F$, $G$, and their derivatives gives point invariants of system (32).

\section{References}

1. Baston, R.J., Eastwood, M.G.: The Penrose Transform. Oxford Science Publications. Oxford University Press, New York (1989)

2. Bryant, R.L., Dunajski, M., Eastwood, M.G.: Metrisability of two-dimensional projective structures. J. Differential Geom. 83(3), 465-499 (2009)

3. Čap, A., Slovák, J.: Parabolic Geometries. I. Mathematical Surveys and Monographs, vol. 154. American Mathematical Society, Providence (2009)

4. Casey, S.: On the Structure of Path Geometries and Null Geodesics in General Relativity. PhD Thesis, University of Cambridge (2013). http://www.damtp.cam.ac.uk/user/md327/stephen_thesis.pdf

5. Casey, S., Dunajski, M., Tod, P.: Twistor geometry of a pair of second order ODEs. Comm. Math. Phys. 321(3), 681-701 (2013)

6. Clifford, W.K.: Mathematical Fragments, Being Facsimiles of his Unfinished Papers Relating to the Theory of Graphs. Macmillan, London (1881)

7. Eastwood, M., Matveev, V.: Metric connections in projective differential geometry. In: Eastwood, M., Miller, W. (eds.) Symmetries and Overdetermined Systems of Partial Differential Equations. The IMA Volumes in Mathematics and its Applications, vol. 144, pp. 339-350. Springer, New York (2008)

8. Egorov, I.P.: Collineations of projectively connected spaces. Doklady Akad. Nauk SSSR (N.S.) 80, 709-712 (1951) (in Russian)

9. Fedorova, A., Matveev, V.S.: Degree of mobility for metrics of Lorentzian signature and parallel (0, 2)-tensor fields on cone manifolds. Proc. London Math. Soc. 108(5), 1277-1312 (2014)

10. Fels, M.E.: The equivalence problem for systems of second-order ordinary differential equations. Proc. London Math. Soc. 71(1), 221-240 (1995)

11. Fulton, W., Harris, J.: Representation Theory. Graduate Texts in Mathematics, vol. 129. Springer, New York (1991)

12. Kiosak, V.A.: Geodesic Mappings of Riemannian Spaces. PhD Thesis, University of Olomouc (2002)

13. Kiosak, V.A., Matveev, V.S., Mikeš, J., Shandra, I.G.: On the degree of geodesic mobility for Riemannian metrics. Math. Notes 87(3-4), 586-587 (2010)

14. Kiosak, V.A., Mikeš, J.: On the degree of mobility of Riemannian spaces with respect to geodesic mappings. In: Bazylev, V.T. (ed.) The Geometry of Imbedded Manifolds, pp. 35-39. Moscow State Pedagogical Institute, Moscow (1986) (in Russian)

15. Kruglikov, B., Matveev, V.: Submaximal metric projective and metric affine structures. Differential Geom. Appl. 33(suppl.), 70-80 (2014)

16. LiE program, software project headed by A.M. Cohen, now maintained by M.A.A. van Leeuwen. Version 2.2.2. http://www-math.univ-poitiers.fr/ maavl/LiE/

17. Liouville, R.: Sur les invariants de certaines équations différentielles et sur leurs applications. Comptes Rendus Hebdomadaires des Séances de l'Académie des Sciences 59, 7-76 (1889)

18. Mettler, T.: Weyl metrisability of two-dimensional projective structures. Math. Proc. Cambridge Philos. Soc. 156(1), 99-113 (2014)

19. Mikeš, J.: Geodesic mappings of affine-connected and Riemannian spaces. J. Math. Sci. 78(3), 311-333 (1996)

20. Nurowski, P.: Projective versus metric structures. J. Geom. Phys. 62(3), 657-674 (2012)

21. Pedersen, H., Tod, K.P.: Three-dimensional Einstein-Weyl geometry. Adv. Math. 97(1), 74-109 (1993)

22. Penrose, R., Rindler, W.: Spinors and Space-Time. I. Cambridge Monographs on Mathematical Physics. Cambridge University Press, Cambridge (1987)

23. Shandra, I.G.: On the geodesic mobility of Riemannian spaces. Math. Notes 68(4), 528-532 (2000)

24. Spencer, D.C.: Overdetermined systems of linear partial differential equations. Bull. Amer. Math. Soc. 75(2), 179-239 (1969)

25. Weyl, H.: The Classical Groups. Princeton University Press, Princeton (1939) 